\title{
Quantum oscillation studies of the Fermi surface of iron-pnictide superconductors
}

\author{
A. Carrington \\ H.H. Wills Physics Laboratory, University of Bristol, Tyndall Avenue, Bristol, BS8 \\ 1TL UK. \\ E-mail: a.carrington@bristol.ac.uk
}

\begin{abstract}
This paper reviews quantum oscillation studies of iron-pnictide superconductors and related materials. These measurements give unique information regarding the full three dimensional topology of the Fermi surfaces and the renormalisation of the quasi-particle masses. The review will cover measurements of the 122 arsenide end members, $\mathrm{XFe}_{2} \mathrm{As}_{2}(\mathrm{X}=\mathrm{Ba}, \mathrm{Sr}, \mathrm{Ca}$ ) which have a spin density wave ground state, but will concentrate on the phosphide end members (LaFePO and $\mathrm{XFe}_{2} \mathrm{P}_{2}$ ) which have a paramagnetic ground state and a Fermi surface topology which is similar to the higher $T_{c}$ superconducting iron-pnictides. All three of the 122 phosphides become superconducting when $\mathrm{P}$ is partially substituted by As, and for the $\mathrm{BaFe}_{2}\left(\mathrm{As}_{1-x} \mathrm{P}_{x}\right)_{2}$ series de Haas-van Alphen oscillations are observable for $0.42 \leq x \leq 1$ with $T_{c}$ up to $25 \mathrm{~K}$. The results show the changes in the Fermi surface topology and the increase in the mass renormalisation as the correlations which induce superconductivity develop.
\end{abstract}

\section{Introduction}

A popular view of the iron-pnictide superconductors is that their unusual properties stem from the unique structure of their Fermi surfaces. Very soon after the discovery of superconductivity at temperatures greater than $40 \mathrm{~K}$, conventional density functional theory (DFT) band-structure calculations using the local density approximation (LDA) (and its derivatives such as the generalised gradient approximation (GGA)) showed that the Fermi surface is mostly composed of small, almost two dimensional, tubes running along the $c$-axis [1]. The tubes at the centre of the Brillouin zone are hole-like whereas those at the corner of the zone are electron-like [see Figs. (11), (15) and (17)]. For the undoped parent materials, for example LaFePO or LaFeAsO, the volume of the hole and electron Fermi surfaces are exactly the same and the material is said to be compensated as the number of electrons and holes are equal.

It was noticed that as these Fermi surfaces are both roughly circular in cross section and as they are the same size, then a single translational vector $\boldsymbol{Q}=(\pi / a, \pi / a)$ maps one surface onto the other. This property, known as nesting, causes a peak in the Lindhard dielectric response function $\chi(\boldsymbol{q})$ at $\boldsymbol{q}=\boldsymbol{Q}$ which can lead to charge or spin density 
wave instabilities depending on the relative strength of the electron-phonon or electronelectron interactions. If the nesting is not so perfect, and assuming electron-electron interactions dominate (as they do for iron-pnictides) then strong spin fluctuations should be expected with wavevectors centred around $\boldsymbol{Q}$. In many models [2, 3, 4, 5] it is these spin-fluctuations which provide the pairing interaction which binds the electrons into Cooper pairs and causes superconductivity. As the Fermi surface geometry is at the heart of this interaction its experimental determination is of high importance.

It should be said before continuing that a completely itinerant electron model, as outlined above, is unlikely to be the whole picture. Although the many body electronelectron correlations are usually assumed to be relatively weak in the iron-pnictides, as compared to the cuprate high $T_{c}$ superconductors for example, they cannot be neglected. For example, according to the DFT calculations the Fermi surfaces of LaFePO and LaFeAsO are very similar, however experimentally LaFePO is a paramagnetic metal which becomes superconducting below $6 \mathrm{~K}$ [6] whereas LaFeAsO has a transition to spin density wave metallic antiferromagnetic state below $130 \mathrm{~K}$ and is not superconducting [7]. An explanation for this difference is to suppose that the magnetic interactions originate primarily from many body electron-electron correlations (for example the $U$ and $J$ parameters in Hubbard type models) but the structure of the spin-fluctuations in momentum space is governed by the Fermi surface geometry [8]. This is backed up by the fact that DFT models generally correctly predict the form of the magnetic order in the static SDW phase. The relative importance of the local interactions and the itinerant behaviour is still under debate.

Given the importance of determining the properties of the Fermi surface it is not surprising that this has been the subject of many theoretical and experimental investigations. By far the most popular and ubiquitous experimental technique is angle resolved photoemission spectroscopy (ARPES). Here the sample is illuminated with photons, typically of energy $6-100 \mathrm{eV}$, and emitted photoelectrons are collected and analysed. As the in-plane momentum of the electrons is conserved when they are emitted, measurements of the energy and momentum of these electrons as a function of angle can be used to deduce the $\varepsilon(\boldsymbol{k})$ relationships for the various bands in the metal. The technique yields a direct 'image' of a 2 dimensional slice through the Fermi surface at a particular $k_{z}$ which is determined by the energy of the photons (with an uncertainty determined by the escape depth of the photoelectrons). One problem with the technique is that it is surface sensitive. The surface electronic structure may differ from the bulk for several reasons. For example, the surface may be charged (and hence effectively doped) if the cleavage plane is not neutral. The technique also has significantly lower resolution than quantum oscillations studies (particularly in $k_{z}$ ).

\subsection{Determining the Fermi surface via quantum oscillations}

Measurements of the Fermi surface properties based on magneto-quantum oscillation (QO) effects are an important complementary technique to ARPES. Although the 
majority of the Fermi surface parameters which may be determined by the QO effects are also accessible by ARPES, the main advantages of QO are: i) it is very insensitive to surface states as it is dominated by the bulk response thus giving an unambiguous probe of the bulk Fermi surface, ii) it has very high $\boldsymbol{k}$-space resolution in all three crystallographic directions - typically Fermi surface cross-sections can be determined to better than $10^{-3}$ of the area of the Brillouin zone, iii) it has very high energy resolution, so that it gives the effective mass very close to the Fermi level $\left(\Delta E \sim \mu_{B} B=0.6 \mathrm{meV}\right.$ for $B=10 \mathrm{~T}$ ). A major limiting factor is that only samples with sufficiently long mean free path will give a measurable signal. High magnetic field and low temperatures are usually essential.

The technique is based on the fact that in a magnetic field the only allowed values of electron momentum $\boldsymbol{k}$ lie on Landau tubes of area $A=\left(n+\frac{1}{2}\right) B / \phi_{0}$, where $\phi_{0}$ is the single electron flux quantum $(h / e)$ and $n$ is an integer known as the Landau index. These two dimensional electron tubes run parallel to the field $B$ and for almost free electrons will have a circular cross-section. As the field is varied the $\boldsymbol{k}$ space radius of a particular Landau level $n$ will increase, and as each level passes through the Fermi surface the states will depopulate giving rise to a saw-tooth like variation of the free energy, periodic in inverse field $1 / B$. The frequency (in inverse field) of the oscillations is related to the $\boldsymbol{k}$-space cross sectional area of the Fermi surface $A_{\boldsymbol{k}}$ by the Onsager relation, $F=\hbar A_{\boldsymbol{k}} / 2 \pi e$. For a three dimensional metal $A_{\boldsymbol{k}}$ will vary as a function of $k_{z}$ and hence each $k_{z}$ slice (assuming $B \| z$ ) will give rise to oscillations of varying frequency and phase. The total signal will be the sum of the contributions from each slice. Usually the sum is dominated by the extremal cross-sections of the Fermi surface where $d A_{\boldsymbol{k}} / d k_{z}=0$ and hence a typical Fermi surface sheet will give rise to a small number of oscillatory signals.

The oscillations in the free energy may be determined by measuring many different physical properties such as the resistivity (Shubnikov-de Haas effect), the magnetisation or magnetic torque (de Haas-van Alphen effect), specific heat etc. Finite temperature, impurity scattering and other effects smear out the discontinuity as the Landau levels pass through $\varepsilon_{F}$. The response may be decomposed into a Fourier series, and the signal is usually dominated by the fundamental harmonic at frequency F. For a three dimensional metal of volume $V$ the full expression for the first harmonic of the oscillatory torque $\mathcal{T}$ is (in SI units)

$$
\begin{aligned}
\mathcal{T} & =\sum_{\text {orbits }} \frac{\partial F}{\partial \theta} \frac{V e^{5 / 2}}{\hbar^{1 / 2} \pi^{2} m_{e}} \frac{B^{3 / 2}}{\left(2 \pi \frac{\partial^{2} A}{\partial k_{\|}^{2}}\right)^{1 / 2}} R_{T} R_{D} R_{S} \sin \left(\frac{2 \pi F}{B}+\phi\right) \\
R_{D} & =\exp \left(-\frac{\pi m_{b}}{e B \tau}\right) \\
R_{T} & =\frac{X}{\sinh X}, \quad X=\frac{2 \pi^{2} k_{B} T m^{*}}{e \hbar B} \\
R_{S} & =\cos \left(\frac{\pi g m_{S}}{2 m_{e}}\right) .
\end{aligned}
$$


This is commonly known as the Lifshitz-Kosevich (LK) formula [9]. The expression includes the most common attenuation factors, $R_{T}, R_{D}$ and $R_{S}$ which are due to finite temperature, impurity scattering and spin-splitting respectively. It should be noted that there are other attenuation factors, due to effects such as mosaic crystal structure or superconductivity which are important in some circumstances. In the expression the quasiparticle mass occurs several times in different forms. $m_{e}$ is the free electron mass and $m_{b}$ is the band mass as calculated from mean-field DFT band structure calculations. The mass $m^{*}$ which occurs in the expression for the temperature dependent damping factor $R_{T}$ is the quasiparticle effective mass renormalized by electron-phonon and electron-electron interactions. $m_{s}$ is the 'spin-mass' which is renormalized by electronelectron interactions (which enhance the Pauli susceptibility) only. The curvature factor, $\left(\frac{\partial^{2} A}{\partial k_{\|}^{2}}\right)^{-1 / 2}$ accounts for the number of states which contribute to the extremal orbit, here $k_{\|}$is the component of $\boldsymbol{k}$ parallel to the field. If $A_{\boldsymbol{k}}$ changes little with $k_{\|}$, for example because the Fermi surface is locally almost two dimensional, then these orbits will have a large intrinsic amplitude.

The Dingle factor, $R_{D}$, is determined by the impurity scattering rate $\tau^{-1}$ and is not normally renormalized by many body factors. For free electrons we can make the substitutions, $m_{b} v_{F}=\hbar k_{F}$, and $\ell=\tau v_{F}$, where $v_{F}$ is the Fermi velocity and $\ell$ is the mean free path, so that

$$
R_{D}=\exp \left(-\frac{\pi \hbar k_{F}}{e B \ell}\right)
$$

For the general case, $\ell$ and $k_{F}$ should be replaced by values averaged over the orbit. A simple approximation is to take $\pi k_{F}^{2}=A=2 \pi e F / \hbar$, so that $R_{D}=\exp (-1140 \sqrt{F} / \ell B)$, with $\ell$ in Angstroms and $F, B$ in Tesla.

This paper will review QO experimental studies of the Fermi surface of the ironpnictide superconductors. Because of the need for high purity samples these studies have mostly been conducted on the stoichiometric end members of the various iron-pnictide series. Charge doping of these parent materials, which is a common route to induce superconductivity, generally induces significant disorder which shortens the mean-freepath to such an extent that $\mathrm{QO}$ are not observable even in the highest available fields $\left(B_{\max } \simeq 70 \mathrm{~T}\right)$. On the other hand, isovalent substitution of As by $\mathrm{P}$ in the series $\mathrm{BaFe}_{2}\left(\mathrm{As}_{1-x} \mathrm{P}_{x}\right)_{2}$ does not induce so much disorder and $\mathrm{QO}$ are observed for dopings producing $T_{c}$ up to $\sim 80 \%$ of the maximum [10].

\section{Parent iron-arsenides in SDW state}

In their undoped state, at ambient pressure, the '1111' (e.g., LaFeAsO) and '122' (e.g., $\mathrm{BaFe}_{2} \mathrm{As}_{2}$ ) iron-arsenides have a spin-density wave ground state. Below a temperature of $140 \mathrm{~K} \mathrm{BaFe}_{2} \mathrm{As}_{2}$ undergoes a structural and magnetic transition (at slightly different temperatures) to a state with stripe ordered magnetic moments with a unit cell which is roughly $\sqrt{2}$ larger (along the edge) and rotated by $45^{\circ}$ compared to the high temperature cell [11]. The structure is actually orthorhombic (Fmmm), with 

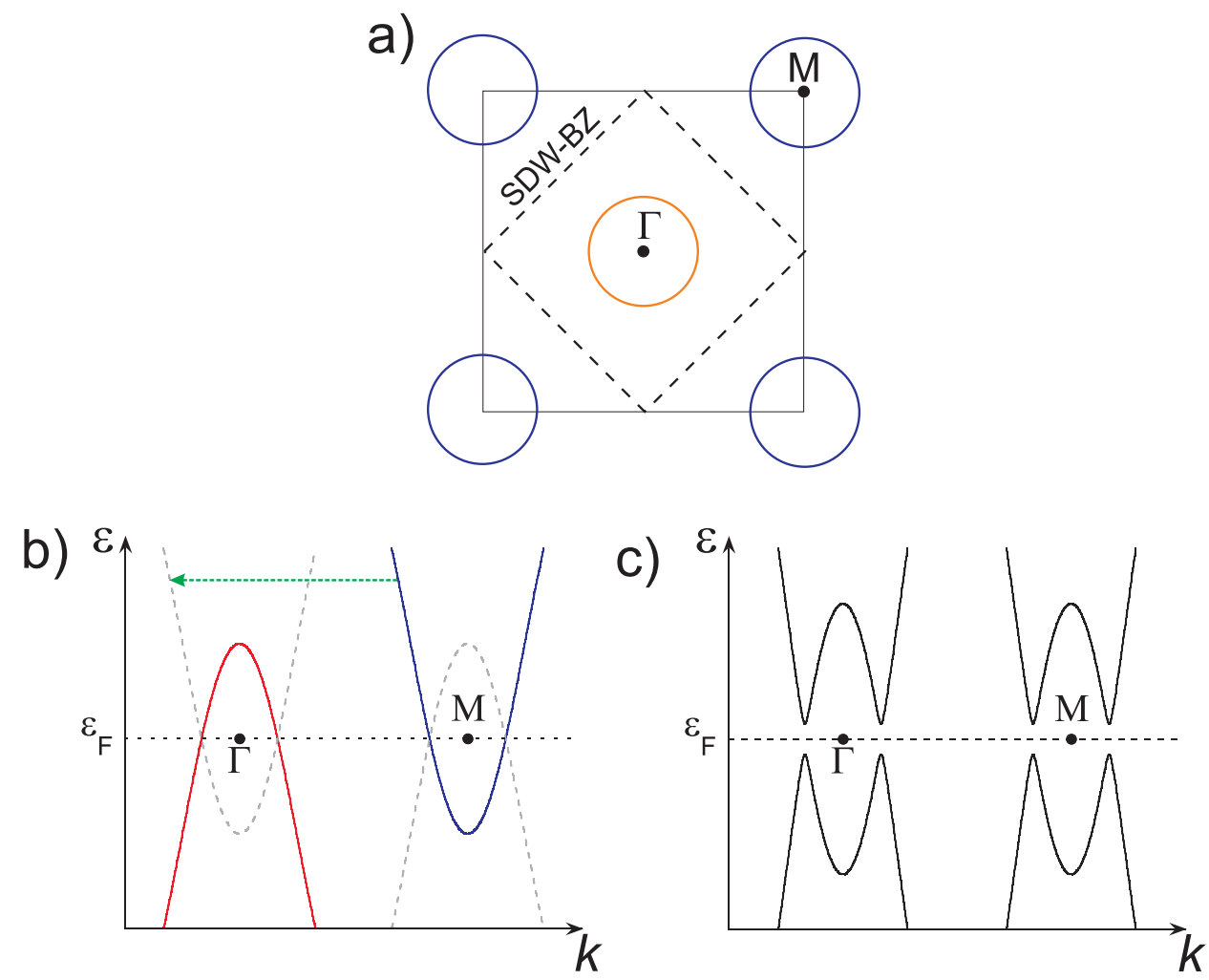

Figure 1. Simplified schematic picture of Fermi surface reconstruction due to band folding at the spin-density wave transition in the iron-pnictides. a) Shows circular Fermi surface sheets, hole like at the zone centre $(\Gamma)$ and electron like at the zone corner $(M)$, in the paramagnetic Brillouin zone (solid line). The change in structure at the SDW transition will cause the Brillouin zone to change (dashed line). b) shows the band-structure energy - momentum curves corresponding to the Fermi surface in a). As the Brillouin zone changes the band at $M$ is translated (folded) back to the $\Gamma$ point in the reduced zone scheme. c) The folded bands hybridize and a gap forms at the Fermi level $\varepsilon_{F}$, and the Fermi surface disappears.

$a=5.6146 \AA, b=5.5742 \AA$, and $c=12.9453 \AA$. The fact that $a$ and $b$ only differ by $0.7 \%$ means that the crystals are highly susceptible to twinning, so that crystals usually have multiple domains in which the $a$ and $b$ directions are switched. Twin free samples have been produced by cooling through the SDW transition with an applied uniaxial stress or a large magnetic field [12, 13, 14].

As a first approximation the effect of this change in structure on the Fermi surface can be understood by a simple band-folding argument. As illustrated in Fig. 1, the change in structure causes the electron band which produced a Fermi surface at the corner of the original cell to fold to the centre of the zone. The electron-like bands and the hole-like bands now cross and in the simple case where the shape and size of hole and electron Fermi surfaces are identical, the bands would cross exactly at the Fermi level. The bands will then hybridize and a gap will appear at the Fermi level, hence the Fermi surface would completely disappear and the material would become insulating. In reality, the original Fermi surface sheets do not have exactly the same in-plane shape 

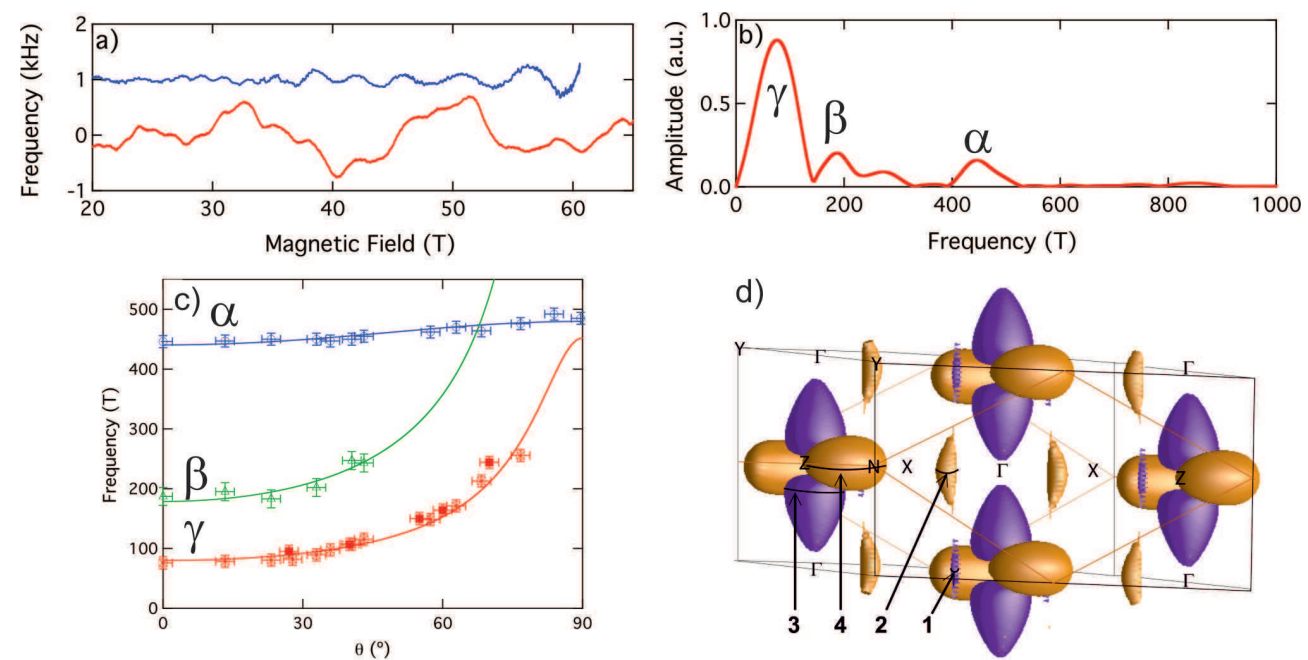

Figure 2. Quantum oscillations in $\mathrm{BaFe}_{2} \mathrm{As}_{2}$. a) Raw oscillator frequency shift which is proportional to the resistance versus field for $B \| c$ and $B \perp c$ (upper and lower curves respectively), b) Fourier transform of the $B \| c$ data. c) Variation of observed SdH peaks with field angle (solid lines are fits to a simple elliptical Fermi surface model). d) DFT fixed moment calculation of the Fermi surface using the negative $U$ procedure to fix the ordered moment. The predicted extremal orbits are labelled 1-4. Figure adapted from Analytis et al. [16].

and there is some corrugation along $k_{z}$, hence the Fermi surface will not be completely wiped out and small three dimensional Fermi pockets will remain. In addition, the formation of the SDW state causes the bands to shift in energy (some bands more than others) so the exact band-structure is not simply related to the folded paramagnetic structure. Fixed moment DFT calculations seem to produce approximately the correct structure - although not the exact Fermi surface topology [15].

Quantum oscillation experiments have been reported on several members of the '122' family $\mathrm{XFe}_{2} \mathrm{As}_{2}$ with $\mathrm{X}=\mathrm{Sr}, \mathrm{Ba}$ and $\mathrm{Ca}$ [17, 16, 18]. Examples of the raw (background subtracted) experimental results for the oscillations in the resistivity of $\mathrm{BaFe}_{2} \mathrm{As}_{2}$ (measured inductively using a tunnel diode oscillator) are shown in Fig. 2. The signals are relatively weak and can only be observed in very high magnetic field (typically greater than $30 \mathrm{~T}$ ). In all these measurements only very low oscillation frequencies were observed. For example for $\mathrm{BaFe}_{2} \mathrm{As}_{2}$, the values with $B \| c$ were reported to be $80 \mathrm{~T}, 190 \mathrm{~T}$ and $440 \mathrm{~T}$, which correspond to $0.3,0.7$ and $1.7 \%$ of the total area of the paramagnetic Brillouin zone respectively [19]. The weak variation of the $\alpha$ frequency with magnetic field angle shows that this comes from a small quasi spherical pocket as anticipated. A DFT calculation of the Fermi surface of $\mathrm{BaFe}_{2} \mathrm{As}_{2}$ based on the observed magnetically ordered unit cell is shown in Fig. 2k). These calculations use the LDA $+\mathrm{U}$ procedure, which is common for strongly correlated metals, with a negative orbital potential $U$ to reduce the ordered moment. This negative $U$ has no physical significance and should be regarded as an empirical fix to the LDA calculations. With moderate shifts of the calculated bands energies $(<60 \mathrm{meV})$, the predicted orbits $1-3$ can be brought into agreement with the 

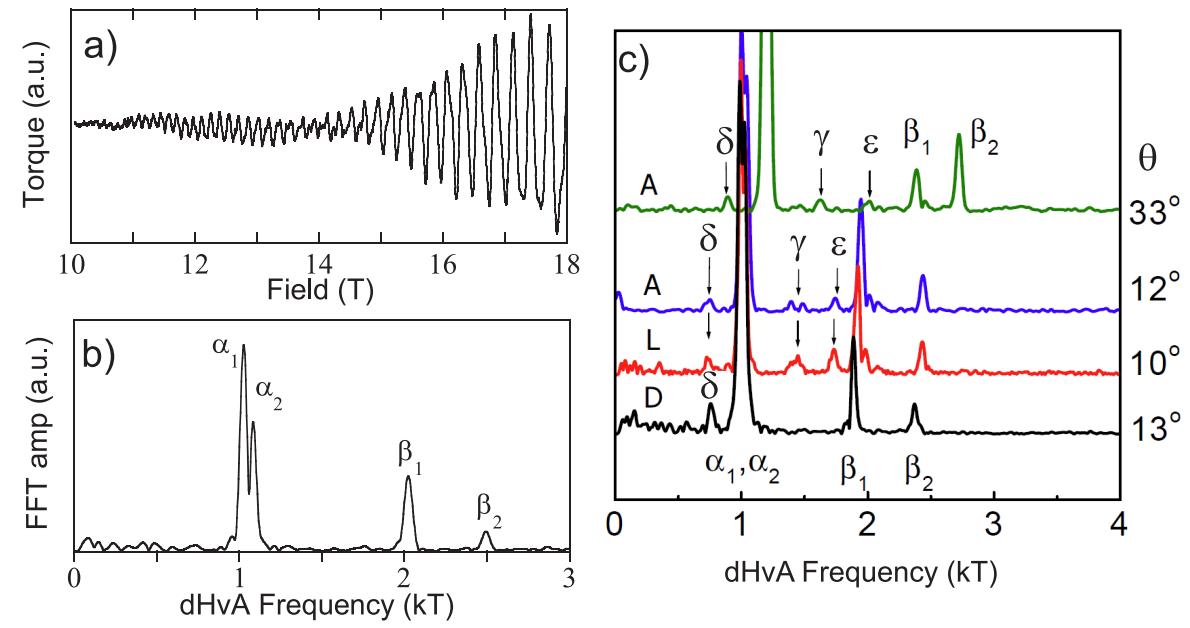

Figure 3. Quantum oscillations in LaFePO. a) Raw torque versus field at $T=0.35 \mathrm{~K}$ with $4^{\text {th }}$ order polynomial subtracted. b) Fast Fourier transform of the same data. c) FFT spectra of different samples (labelled A, L D) at several angles $\theta$ showing the various extremal orbit peaks. Figure adapted from data in Ref. [21].

experimental results in Ref. [16]. In this study the Fermi surface determination was incomplete as all the predicted orbits were not observed experimentally.

Recently a complete Fermi surface determination of de-twinned $\mathrm{BaFe}_{2} \mathrm{As}_{2}$ was reported [20]. In these detwinned samples the oscillations are large in amplitude even in low fields less than $10 \mathrm{~T}$. All extremal orbits of the calculated Fermi surface were observed, and the calculations could be brought into almost exact agreement with experiment with appropriate band energy shifts $(<65 \mathrm{meV})$. The determined Fermisurface is quite similar that shown in Fig. 2 but has some differences in detail for some of the pockets.

\section{3. $\mathrm{LaFePO}$}

The 1111 compound LaFePO was the first discovered iron-pnictide superconductor [6] and was also the first paramagnetic iron-pnictide in which QO were observed [21]. Unlike the isomorphic arsenide $\mathrm{LaFeAsO}, \mathrm{LaFePO}$ is superconducting in its stoichiometric state (some reports have suggested that a few percent of oxygen vacancies are needed to induce superconductivity [22, 23], however, it is not clear if this is necessary [21]). Unlike LaFeAsO, LaFePO is not magnetically ordered and magnetism can not be induced by small amounts of doping away from stoichiometry. The superconducting transition temperature is quite low $T_{c} \simeq 6 \mathrm{~K}$ and the upper critical fields $\left(H_{c 2} \| c=0.68 \mathrm{~T}\right.$, $\left.H_{c 2} \perp c=7.2 \mathrm{~T}\right)$ are easily exceeded with conventional superconducting magnets [24]. It is therefore an ideal candidate for study by quantum oscillations effects.

Data were first reported by Coldea et al [21], closely followed by Sugawara et al.25. As the best available single crystals of LaFePO are quite small (typically planar dimensions of a few hundred microns and thickness a few tens of microns) the 


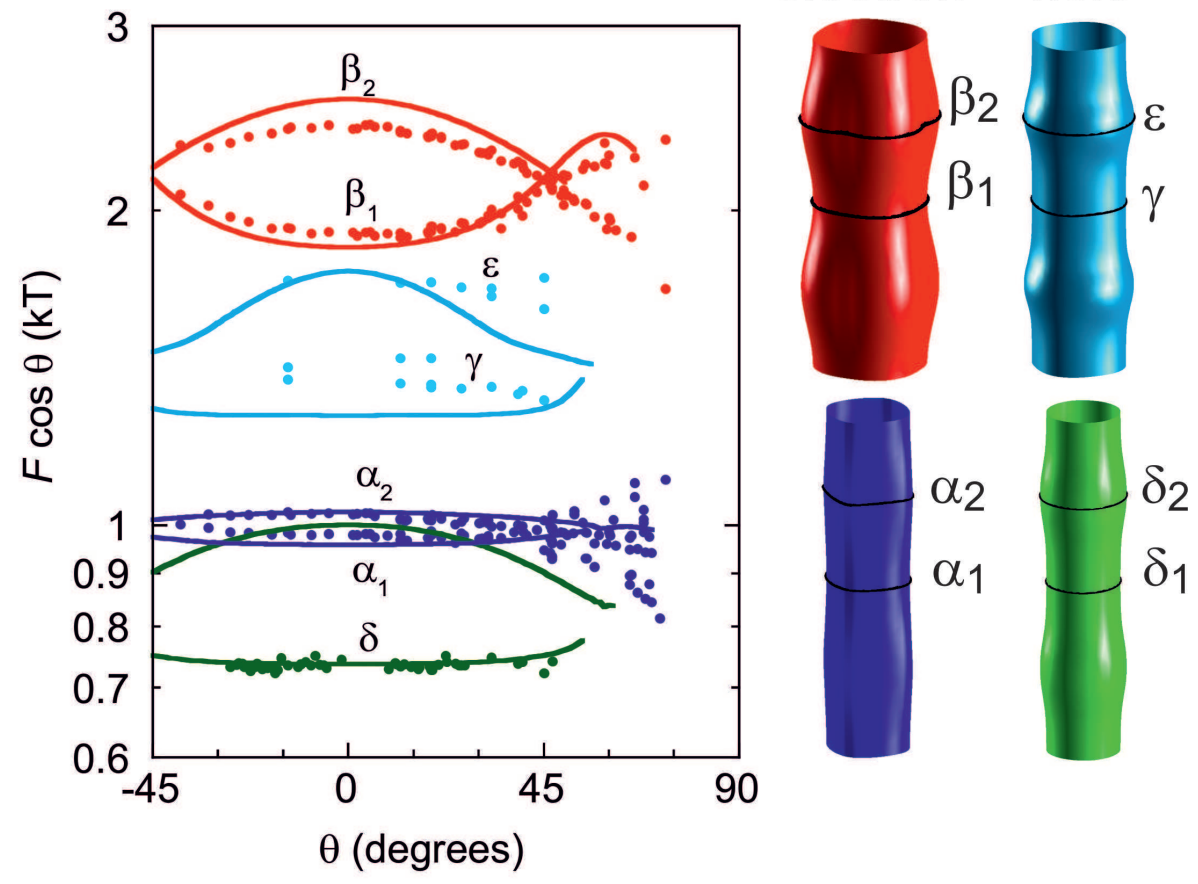

Figure 4. Observed dHvA oscillation frequencies in LaFePO (solid symbols) versus field angle as the field is rotated from $B \| c\left(\theta=0^{\circ}\right)$ to $B \perp c$. The values derived from the DFT calculation, after shifting the band energies (as described in the text) are shown by the solid lines. The right panel shows the extremal orbits on the electron and hole Fermi surface sheets. Figure adapted from data in Carrington et al [24].

microcantilever torque method is well suited. Examples of raw torque versus magnetic field data for this compound are shown in Fig. 3, The data are best appreciated by calculating the fast Fourier transform (with respect to inverse field). A total of 7 fundamental frequencies are observed plus harmonics of the strongest amplitude signals ( $\alpha$ and $\beta$ orbits). Measurements were repeated as a function of field angle as the field was rotated from parallel to the $c$-axis $\left(\theta=0^{\circ}\right)$ towards the $a b$-plane. As the field is rotated the extremal electron orbits transverse different sections of the Fermi surface, and hence by analyzing the field angle dependence of the dHvA frequencies, $F(\theta)$, a detailed model of the Fermi surface can be constructed.

For a perfectly two dimensional Fermi surface, $F$ is inversely proportional to $\cos \theta$ and hence by plotting $F \cos \theta$ versus $\theta$ small deviations from two dimensionality can be easily seen. The simplest distortion of a two dimensional Fermi surface in a tetragonal system is a cosine dispersion, where the $\varepsilon(k)=\hbar^{2}\left(k_{x}^{2}+k_{y}^{2}\right) / 2 m+t_{\perp} \cos \left(c k_{z}\right)$. In this case, there are two extremal frequencies, the minimum $F_{\min }$ and the maximum $F_{\max }$, which vary with angle according to the Yamaji formula [26]

$$
F_{\min , \max }(\theta) \cos \theta=F_{a v} \pm \Delta F J_{0}\left(c k_{F} \tan \theta\right) .
$$

In this expression, the first term is the mean frequency $\left(F_{a v}=F_{\min }+F_{\max }\right) / 2$ at $\theta=0$, the prefactor of the second term is $\Delta F=\left(F_{\max }^{0}-F_{\min }^{0}\right) / 2, J_{0}$ is the Bessel function 


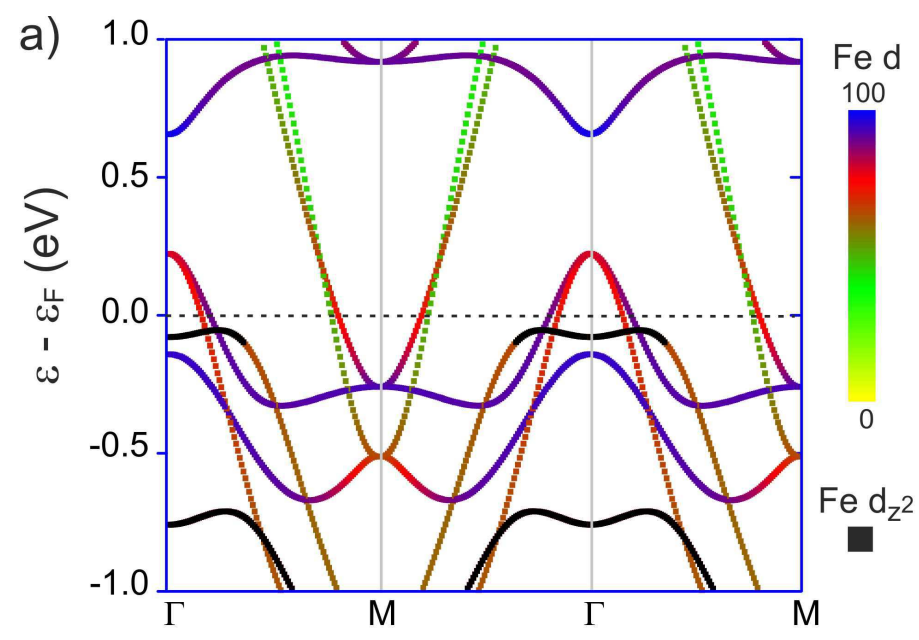

b)
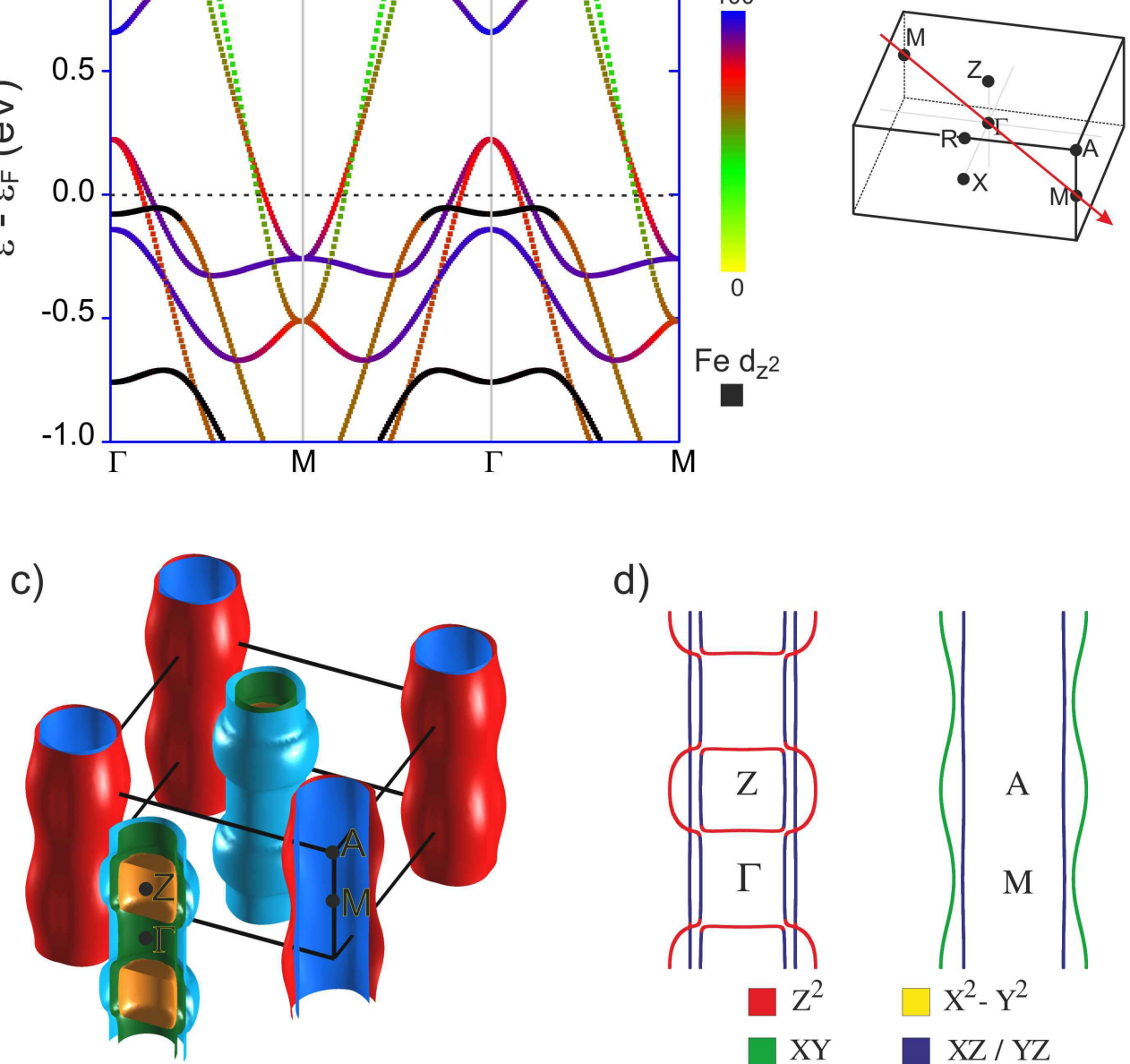

d)
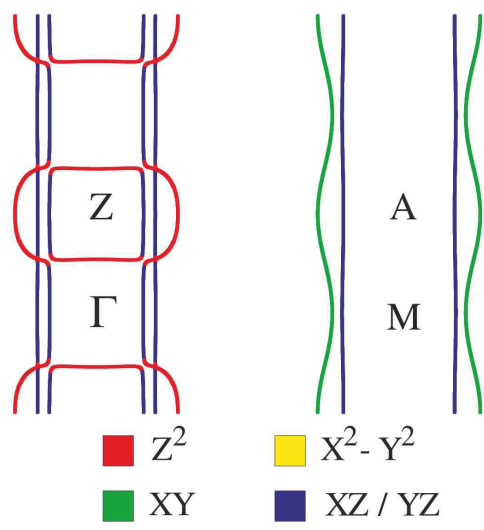

Figure 5. Calculated electronic structure of $\mathrm{LaFePO}$ without spin orbit interaction or adjustment to fit experimental data. a) Band structure energy-momentum along the zone diagonal. The percentage of Fe- $d$ character is indicated by the colour scale, with the band with predominant $\mathrm{Fe} d_{z^{2}}$ character overprinted in black. b) Brillouin zone with symmetry labels. c) Three dimensional Fermi surface. d) $c$ axis (110) slice through the Fermi surface with predominant band character indicated by the colour scale. Figure adapted from Ref. [24].

and $k_{F}^{2}=2 e F_{a v} / \hbar$. At certain angles $J_{0}=0$ and $F_{\min }=F_{\max }$ and here all parts of the Fermi surface interfere constructively to give a large increase in the amplitude of the oscillations. Such a point is observed at $\theta=42(2)^{\circ}$ for the $\beta$ frequencies of LaFePO, which together with the overall behaviour of $F_{\min }$ and $F_{\max }$ are very strong evidence that they originate from a single $c$-axis warped section of Fermi surface (see Fig. (4). The two $\alpha$ frequencies are much closer indicating that they come from an almost two dimensional section of Fermi surface with little warping, and no clear Yamaji amplitude peak is seen. For the lowest frequency $\delta, F \cos \theta$ shows almost no change with $\theta$, consistent with it coming from an almost 2D Fermi surface section. The remaining two frequencies $\varepsilon$ and $\gamma$ are much weaker; they were only observed above $B=30 \mathrm{~T}$ and again $F \cos \theta$ shows minimal change with $\theta$ within the noise. 

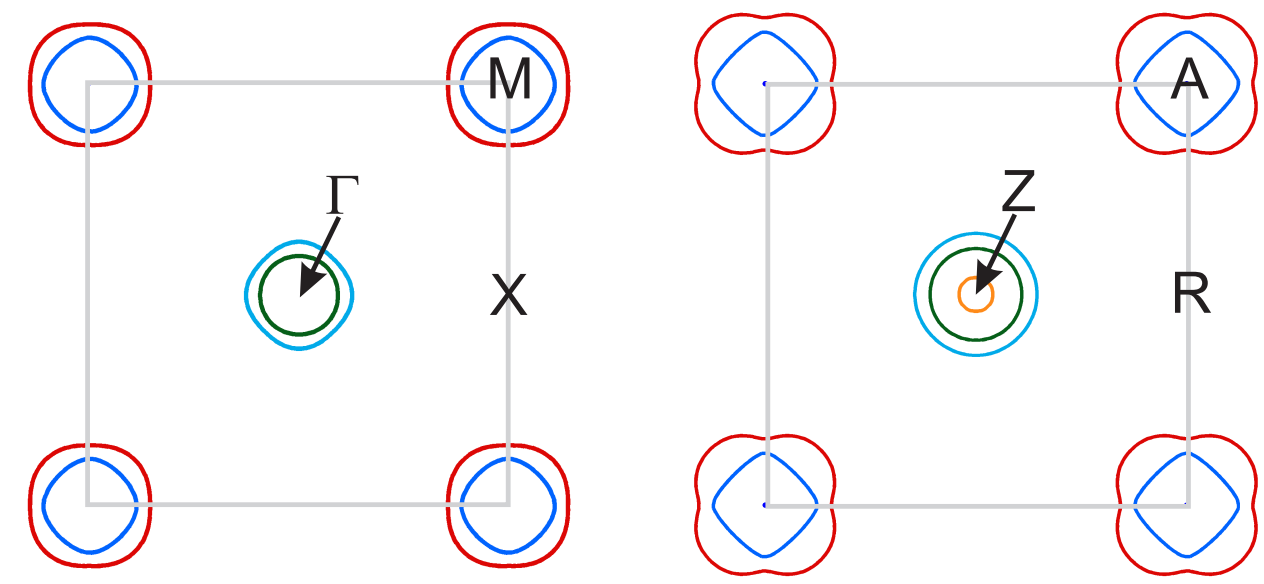

Figure 6. In plane cross sections of the calculated Fermi surface of LaFePO including the spin-orbit interaction and with bands shifted to best match the dHvA data. Figure adapted from Ref. 24].

Further analysis of the data is greatly aided by band-structure calculations. The experimental dHvA frequency versus field angle data can be compared to these calculations and adjustments made to best fit the data. Often this can be accomplished with a small number of parameters, usually a single energy shift for each band or group of bands. The alternative is to fit the data to a series of appropriate geometrical harmonics. This latter approach works well when the Fermi surface is relatively simple and only has small deviations from a sphere or a two dimensional tube and only a small number of harmonics are required (for example, $\mathrm{SrRu}_{2} \mathrm{O}_{4}$ [27] or $\mathrm{Tl}_{2} \mathrm{Ba}_{2} \mathrm{CuO}_{6+\delta}$ [28, 29]).

For LaFePO the band-structure calculations were done [21, 24] using the augmented plane wave plus local orbitals methods as implemented in the WIEN2K code [30]. For these calculations the experimental lattice parameters and internal coordinates were used. A plot of the energy-momentum band structure along the $\Gamma-M$ direction (Fig. (5)) shows two hole bands crossing the Fermi level close to $\Gamma$ and two electron bands crossing close to $M$. So apart from the doubling of bands the detailed band-structure looks quite similar to the simplified picture in Fig. 1, There are however two complications. First the outer electron surface has significant $c$-axis and in-plane warping and second, there is a fifth band, mostly coming from the Fe $d_{z^{2}}$ orbital which cuts across the two dimensional hole bands and forms a closed three dimensional pocket close to $Z$.

The assignment of the $\alpha$ and $\beta$ orbits to the inner and outer electron sheets can be made rather easily. The calculation fairly accurately predicts the size and warping of these sheets. The relatively strong $c$-axis warping of the $\beta$ electron sheet and the much more two dimensional nature of the $\alpha$ electron sheet is well reproduced. To get exact agreement with the data it is necessary to shift the calculated electron bands up in energy ( $+85 \mathrm{meV}$ and $+30 \mathrm{meV}$ for the $\alpha$ and $\beta$ orbits respectively). The physical meaning of these band shifts will be discussed later. With this adjustment the agreement is very good. The calculated $c$-axis warping of the $\beta$ sheet is slightly too large: experimentally 
$\Delta F / F_{a v}=0.23$ whereas the calculation gives $\Delta F / F_{a v}=0.33$, but for the $\alpha$ sheet it is much closer. Without the inclusion of spin-orbit coupling the two electron bands are degenerate along the zone edges $X-M$ and $R-A$. This degeneracy is lifted by the spin-orbit interaction and a gap of $\sim 50 \mathrm{meV}$ appears in the calculations. As the two electron bands need to be shifted by different amounts to get agreement with experiment this implies that this gap is actually about double this (i.e., around $100 \mathrm{meV}$ ). This gap can be seen on the cross sections through the fitted Fermi surfaces shown in Fig. 6.

Identification of the hole orbits is difficult. The lowest observed frequency $\delta$ is likely the minimum of the inner hole sheet which is centred at $\Gamma$. As predicted by the calculation there is minimal change in $F \cos \theta$ for this orbit as the sheet is locally close to two dimensional at this point. It is brought into perfect alignment by shifting all the hole bands down by $53 \mathrm{meV}$. In that case the maximum of the inner hole band almost perfectly coincides with the inner electron band $\alpha$. The assignment of the other two frequencies $\gamma$ and $\varepsilon$ is more problematic. With the above mentioned shift of the three hole band energies it can be seen (Fig. (4) that $\gamma$ and $\varepsilon$ approximately correspond to the minimum and maximum of the largest hole sheet. The variation of $\varepsilon$ with $\theta$ however is much less than expected. As these orbits are only observed at high magnetic field $(>30 \mathrm{~T})$ it is possible that either or both of them result from magnetic breakdown or torque interaction effects arising from the electron orbits [24]. In the calculations, the energy of the Fe $d_{z^{2}}$ band, which gives rise to the warping of the hole sheets and the small spherical pockets at $Z$, varies very strongly with the height of the $\mathrm{P}$ atom and so it is possible that it does not actually cross the Fermi level. In this case the above rigid band shifting will not work accurately because the $d_{z^{2}}$ band has crossed and hybridized with the two dimensional $d_{x z \| x y}$ bands. Instead, we might expect the hole bands are almost perfectly two dimensional, thus giving rise to only two dHvA frequencies with no variation in $F \cos \theta$ as a function of $\theta$. $\delta$ is almost certainly from the inner hole band but which of $\gamma$ and $\varepsilon$ is from the largest hole band is uncertain.

The effective mass of the quasiparticles on each of the orbits was deduced by measuring the temperature dependence of the dHvA oscillation amplitudes and fitting this to the expression for $R_{T}$ (Eq. (1)). The masses for the electron orbits range from 1.7 to $1.9 \mathrm{~m}_{e}$. This should be compared to the masses calculated from the bandstructure (for the shifted bands) which range from 0.7 to $0.9 m_{e}$, hence the many-body mass enhancement factors $m^{*} / m_{b}-1=\lambda$ range from 1.0(2) to 1.7(2) for the 4 orbits (see Table 1). The correlation is much stronger than expected from electron-phonon scattering $\lambda_{e p} \simeq 0.21$ [31, and likely results from electron-electron interactions. ARPES measurements on LaFePO concluded that the bands were all narrowed by a factor $\sim 2.2$, hence this implies that the electron correlations act over a wide energy range rather than being localised close to the Fermi level. This goes against interactions with low energy spin fluctuations being the major cause of the mass enhancement. The masses of the hole orbits are quite similar, however the errors are larger on account of the smallness of the signals. It is unclear at present whether the masses of the observed orbits are sufficient to account for the measured electron specific heat $\gamma_{e}$. Four two dimensional Fermi 

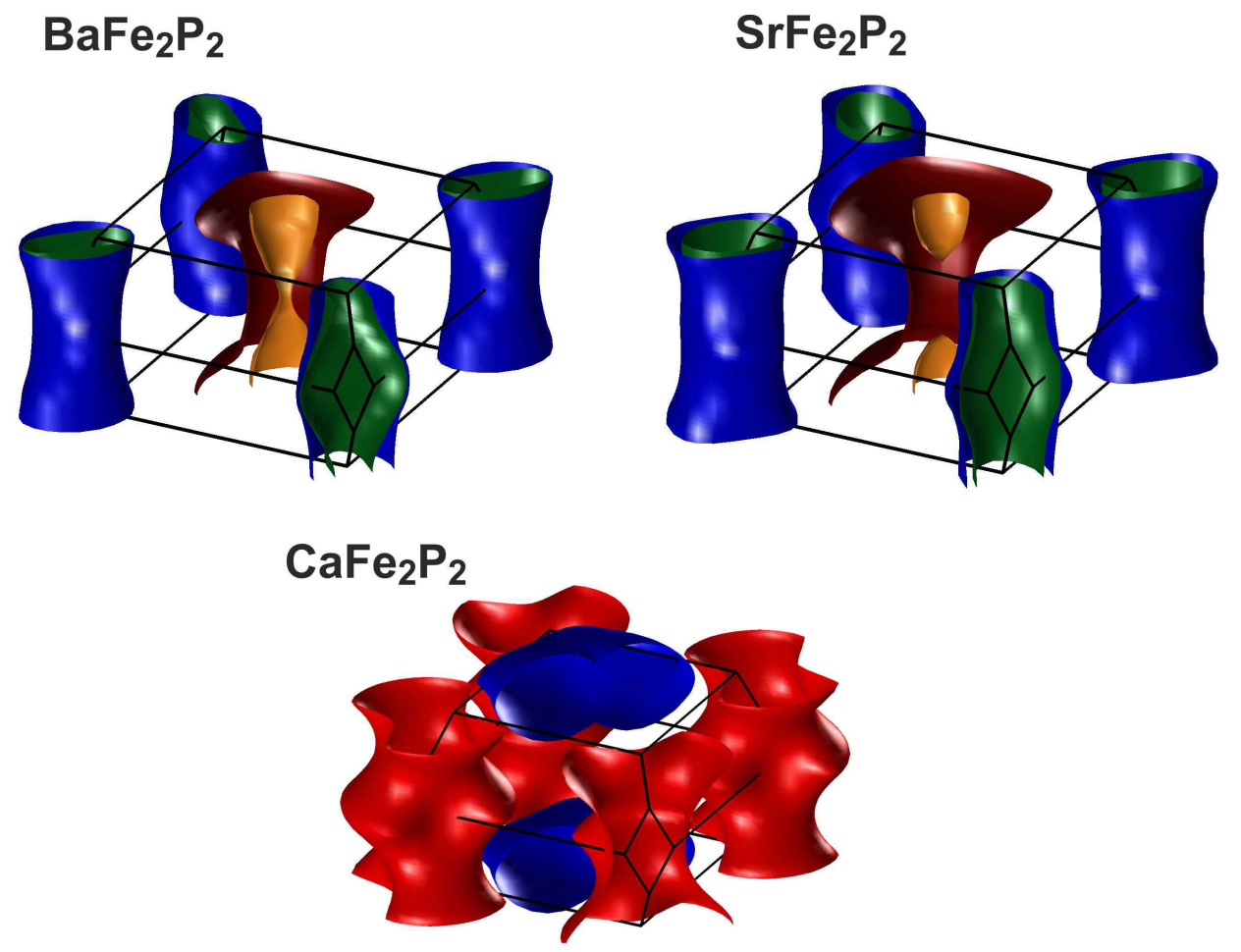

Figure 7. Fermi surfaces of the 122 iron phosphides, $\mathrm{BaFe}_{2} \mathrm{P}_{2}, \mathrm{SrFe}_{2} \mathrm{P}_{2}$, and $\mathrm{CaFe}_{2} \mathrm{P}_{2}$, determined by DFT calculations with bands shifted to best match the dHvA measurements [36, 37, 38].

surfaces each with $m^{*} / m_{e}=2$ would give $\gamma_{e}=6 \mathrm{~mJ} \mathrm{~mol}^{-1} \mathrm{~K}^{-2}$ whereas the reported value on polycrystalline samples was $\gamma_{e}=12.7 \mathrm{~mJ} \mathrm{~mol}^{-1} \mathrm{~K}^{-2}[22]$. This discrepancy could indicate that a heavy hole sheet $\left(m^{*} / m_{e} \simeq 11\right)$ has not yet been detected but the heat capacity measurement also needs to be checked on single crystal samples.

\section{122 phosphides: $\mathrm{BaFe}_{2} \mathbf{P}_{2}, \mathrm{SrFe}_{2} \mathbf{P}_{2}, \mathrm{CaFe}_{2} \mathbf{P}_{2}$}

Studies of the Fermi surfaces of the 122 phosphides are important for a number of reasons. Unlike the corresponding arsenides, the Fermi surfaces of the phosphides are very similar to those of the superconducting phases of the Ba and $\mathrm{Sr} 122$ materials. In all three (Ba, Sr, Ca) phosphides superconductivity can be induced by partial substitution of $\mathrm{P}$ with isovalent As [32, 33] and hence the pure phosphide can be viewed as the end member of these series. The maximum $T_{c}$ produced by $\mathrm{P} / \mathrm{As}$ substitution is very comparable to that obtained by the non-isovalent dopants K [11] / Co [34] or pressure [35].

The 122 phosphides are all paramagnetic, non-superconducting metals. Calculations of the Fermi surface of the $\mathrm{Ba}$ and $\mathrm{Sr}$ phosphides closely resemble those of the corresponding arsenides in their paramagnetic state (i.e., above $T_{\mathrm{SDW}}$ ). The Fermi surface of $\mathrm{CaFe}_{2} \mathrm{P}_{2}$ on the other hand, closely resembles that calculated for $\mathrm{CaFe}_{2} \mathrm{As}_{2}$ in its high pressure state. One effect of replacing As by $\mathrm{P}$ in the 122 materials is to decrease 

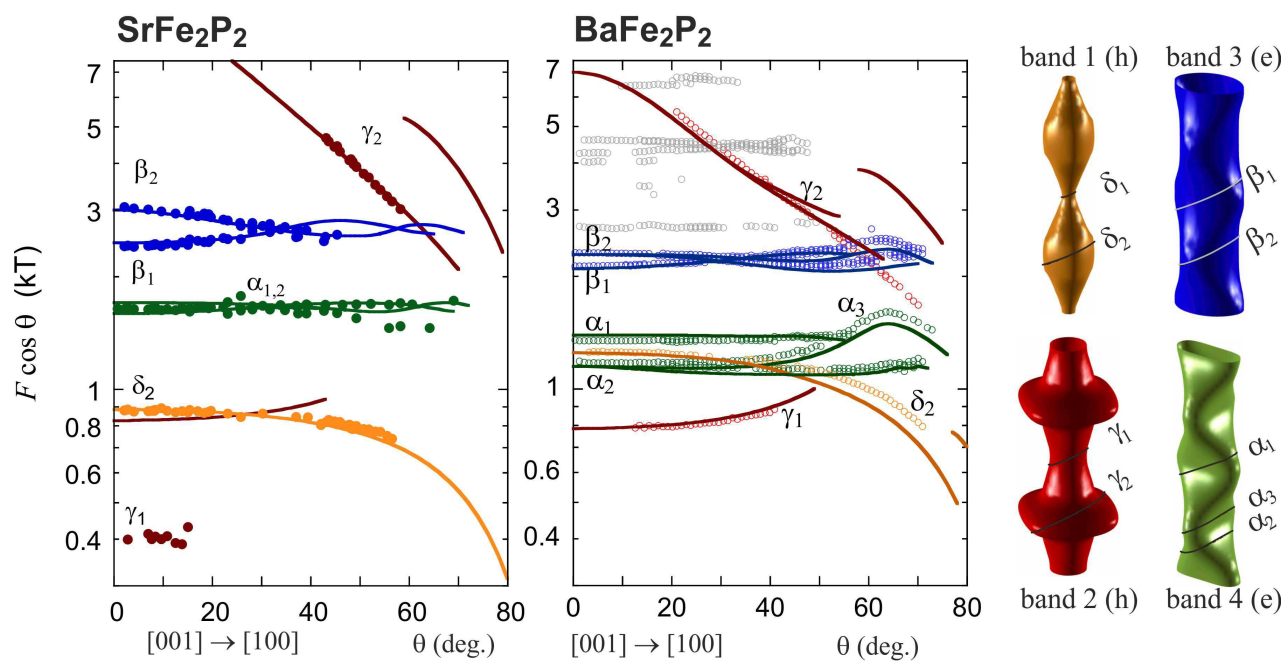

Figure 8. Experimental dHvA frequencies versus magnetic field angle for rotations from $B \| c$ [001] to $B \| a[100]$ for both $\mathrm{SrFe}_{2} \mathrm{P}_{2}$ [36] and $\mathrm{BaFe}_{2} \mathrm{P}_{2}$ [38] (symbols). The unlabelled points in grey in the $\mathrm{BaFe}_{2} \mathrm{P}_{2}$ panel are harmonics of the $\alpha$ and $\beta$ orbits. The orbit labels for $\mathrm{SrFe}_{2} \mathrm{P}_{2}$ have been changed from that in Ref. 36] to be consistent with those used for $\mathrm{BaFe}_{2} \mathrm{P}_{2}$. The solid lines are the frequencies calculated from the DFT calculations with shifted bands. The far right panel depicts the locations of the various orbits on the Fermi surface of $\mathrm{BaFe}_{2} \mathrm{P}_{2}$. The labels also apply to $\mathrm{SrFe}_{2} \mathrm{P}_{2}$ which has a very similar Fermi surface (see Fig. [7). Figure adapted from Refs. 36] and 38]

the distance between the Fe planes and hence increase the pnictogen-pnictogen bonding. In the case of $\mathrm{CaFe}_{2} \mathrm{As}_{2}$ the application of pressure also decreases the interplane distance and for $P>0.35 \mathrm{GPa}$ there is sudden, very large $(\sim 10 \%)$ decrease in the $c$-axis length. This high pressure phase is known as the collapsed tetragonal state. $\mathrm{CaFe}_{2} \mathrm{P}_{2}$ has a similar interplane distance as the collapsed tetragonal state of $\mathrm{CaFe}_{2} \mathrm{As}_{2}$ and hence a similar Fermi surface. The calculated Fermi surfaces for all three compounds are shown in Fig. 7.

dHvA studies have been completed for all three of these 122 phosphides [36, 37, 10, 38]. The oscillations can be observed down to low field $(\sim 5 \mathrm{~T})$ and clear dHvA peaks in the Fourier spectra are observed for orbits on all four sheets of Fermi surface. Experimental dHvA frequencies versus magnetic field angle for both $\mathrm{SrFe}_{2} \mathrm{P}_{2}$ [36] and $\mathrm{BaFe}_{2} \mathrm{P}_{2}$ [38] are shown in Fig. 8. For $\mathrm{SrFe}_{2} \mathrm{P}_{2}$ by far the largest signal comes from the $\alpha$ (inner electron) orbits, followed by the $\beta$ (outer electron) orbits. The large $\alpha$ orbit signal originates from the fact that the in-plane area of this orbit changes very little with $k_{z}$ and hence the curvature factor (Eq. 22) is large. This is demonstrated by the flat behaviour of $F_{\alpha} \cos (\theta)$ in Fig. 8. It should be noted however, that in this case, this does not mean that this sheet of Fermi surface is highly two dimensional. Because of the screw symmetry of the body centred tetragonal structure of the 122 materials, the in-plane elliptical shape of the Fermi surface at the corner of the zone rotates by $\pi / 2$ between 
Table 1. Mass enhancement factors $\left(\lambda=m^{*} / m_{b}-1\right)$ in iron-phosphides. Here $m^{*}$ is the measured thermal mass and $m_{b}$ is the DFT band structure mass at the same angle for with the band shifted to best match the observed dHvA frequency. For Ba-122, Sr-122 and LaFePO the orbit labels are as in Figs. (8) and (4). For Ca-122 the hole orbit labelled here as $\delta_{2}$ is the $\beta$ orbit in Fig. (10).

\begin{tabular}{lllll}
\hline & $\mathrm{LaFePO}$ & $\mathrm{BaFe}_{2} \mathrm{P}_{2}$ & $\mathrm{SrFe}_{2} \mathrm{P}_{2}$ & $\mathrm{CaFe}_{2} \mathrm{P}_{2}$ \\
Electron $\alpha_{1}$ & 1.3 & 0.8 & 1.1 & 0.5 \\
Electron $\alpha_{2}$ & 1.6 & 0.9 & 0.9 & 0.5 \\
Electron $\beta_{1}$ & 1.0 & 0.7 & 0.6 & - \\
Electron $\beta_{2}$ & 1.1 & 0.8 & 0.6 & - \\
Hole $\delta_{1}$ & 1.4 & - & - & - \\
Hole $\delta_{2}$ & - & 0.8 & 0.7 & 0.5 \\
Hole $\gamma_{1}$ & - & 0.6 & 0.5 & - \\
Hole $\gamma_{2}$ & - & 0.6 & 0.7 & - \\
\hline
\end{tabular}

the centre and top of the zone (see Fig. 9). This type of distortion has been described as resembling a snake that has swallowed a chain [27]. Exactly half way up the zone, this odd symmetry $\cos c k_{z} \sin 2 \phi$ term $\left(k_{2,1}\right.$ in the notation of a cylindrical harmonic expansion [27, 29]) is zero, and the shape is closest to circular (even symmetry cosine distortion terms remain) (see Fig. 91). The anisotropy of the individual Fermi surface sheets, parameterized by the anisotropy of the plasma frequencies (which in the isotropic scattering rate limit is equal to the square-root of the resistance anisotropy) are shown in Table 2,

For $\mathrm{SrFe}_{2} \mathrm{P}_{2}$ the calculated $(\alpha / \beta)$ electron Fermi surfaces are brought into almost perfect alignment with the data by shifting them up by $49 \mathrm{meV}$ and $59 \mathrm{meV}$ respectively. The outer hole sheet in this material has very strong warping close to the top of the zone which as in LaFePO is caused by the band acquiring strong $\mathrm{Fe} d_{z}^{2}$ character. At an angle of $\theta \sim 50^{\circ}$ the large warped sections of Fermi surface are almost parallel and so there the curvature factor strongly increases. Hence, it is close to this angle where this orbit is most easily observed. Interestingly, the calculation correctly predicts the size of this part of this Fermi surface without any band energy shifting necessary. As will be discussed below this likely is because this Fermi surface section is does not nest with any of the electron sheets. The inner hole sheet is somewhat smaller than the electron sheets and is shifted down in energy by $110 \mathrm{meV}$ compared to the DFT calculation. This causes the sheet to pinch off (see Fig. 7), so $\delta_{1}$ in Fig. 8 ceases to exist.

An important check of the band shifting fitting of the DFT calculations to the dHvA data is to calculate the volume of the Fermi surface sheets. These materials are all expected to be compensated metals, so they should have equal numbers of electrons and holes. For $\mathrm{SrFe}_{2} \mathrm{P}_{2}$ with the shifts, the $\delta, \gamma, \alpha$ and $\beta$ bands contain $-0.026,-0.284$, 0.108 and 0.197 hole/electrons respectively, and so the charge balance remains almost exact. The imbalance of 0.005 extra holes per unit cell is equivalent to $<2 \%$ of the 

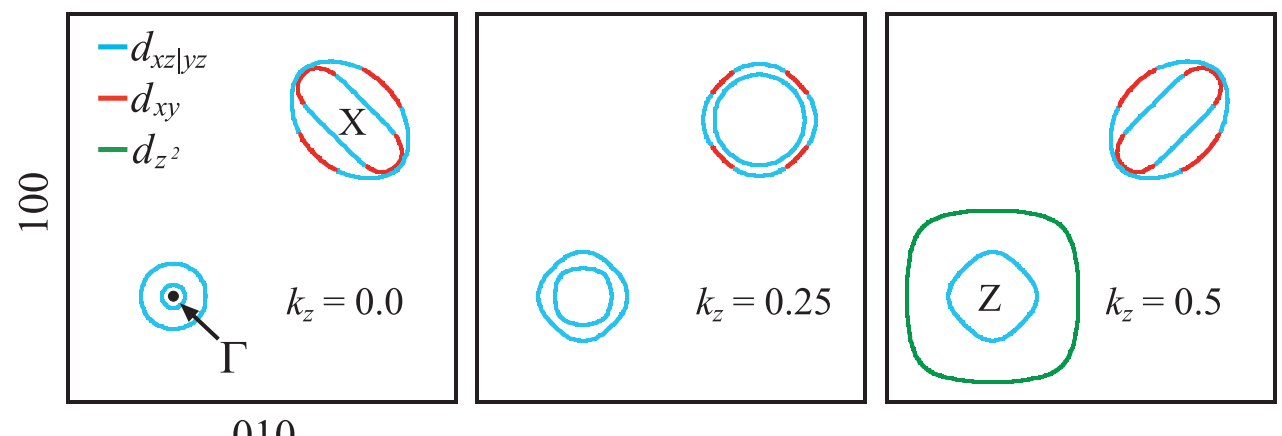

Figure 9. Cross-sections of the determined Fermi surface of $\mathrm{BaFe}_{2} \mathrm{P}_{2}$ in the $a b$ plane at three different $k_{z}$ values (quoted in units of $c^{*}$ ). The strongest band character at each $k$-point is indicated. Note that the zone corner is labelled $\mathrm{X}$ in the body centred unit cell of the 122 compounds. This is equivalent to $\mathrm{M}$ in the simple tetragonal unit cell of LaFePO. Figure adapted from Ref. 38 .

Table 2. Calculated in-plane $\left(\omega_{p, a}^{*}\right)$ and $c$-axis $\left(\omega_{p, c}^{*}\right)$ plasma frequencies in $\mathrm{eV}$ units using the bands shifted to best fit the dHvA data. The values have been renormalized by factor $\left(m^{*} / m_{b}\right)^{-1 / 2}$ where $m^{*} / m_{b}$ is the average mass enhancement for the particular sheet (see Table 11). The anisotropy $\Gamma=\omega_{p, a}^{*} / \omega_{p, c}^{*}$, which in the isotropic scattering approximation is the square root of the conductivity anisotropy, is also given for each Fermi surface sheet. For LaFePO the possible contribution of the small spherical $d_{z^{2}}$ sheet has been ignored. The anisotropy of the remaining two hole sheets is a lower bound as all orbits have not clearly yet been identified.

\begin{tabular}{lllllllllllllll}
\hline & \multicolumn{3}{c}{ LaFePO } & \multicolumn{3}{c}{$\mathrm{BaFe}_{2} \mathrm{P}_{2}$} & \multicolumn{4}{c}{$\mathrm{SrFe}_{2} \mathrm{P}_{2}$} & \multicolumn{4}{c}{$\mathrm{CaFe}_{2} \mathrm{P}_{2}$} \\
& $\omega_{p, a}^{*}$ & $\omega_{p, c}^{*}$ & $\Gamma$ & $\omega_{p, a}^{*}$ & $\omega_{p, c}^{*}$ & $\Gamma$ & $\omega_{p, a}^{*}$ & $\omega_{p, c}^{*}$ & $\Gamma$ & $\omega_{p, a}^{*}$ & $\omega_{p, c}^{*}$ & $\Gamma$ \\
Hole $(\delta)$ & 0.55 & 0.06 & 8.7 & 0.68 & 0.28 & 2.4 & 0.54 & 0.26 & 2.1 & 1.50 & 2.78 & 0.5 \\
Hole $(\gamma)$ & 0.55 & 0.09 & 6.2 & 1.02 & 1.06 & 1.0 & 1.22 & 1.70 & 0.7 & & & \\
Elec $(\alpha)$ & 0.66 & 0.03 & 26 & 0.98 & 0.42 & 2.3 & 1.28 & 0.25 & 5.2 & 1.86 & 1.42 & 1.3 \\
Elec $(\beta)$ & 1.04 & 0.20 & 5.3 & 1.32 & 0.25 & 5.3 & 1.27 & 0.40 & 3.2 & & & \\
\hline
\end{tabular}

total volume of the hole sheets.

The data for $\mathrm{BaFe}_{2} \mathrm{P}_{2}$ are in many ways quite similar to those for $\mathrm{SrFe}_{2} \mathrm{P}_{2}$. The general shape of all four sheets of Fermi surface are very similar and also the absolute sizes are quite comparable. The most significant difference is the size of the inner hole sheet, particularly orbit $\delta_{2}$. The cross section of this sheet is almost identical to the $\alpha$ electron sheet. In fact, the frequency $\delta_{2}$ is right between the two extremities of that from the $\alpha$ sheet. This suggest the possibility that there could be almost perfect nesting between these electron and hole sheets. As mentioned above, however the electron sheets have quite elliptical cross-sections at the centre and top of the zone so although the areas match the shape does not. However, half way between these two extremities the electron sheet becomes almost circular and therefore matches very well the hole sheet (see Fig. 9). In addition the band-character of these sheets is also very similar. It should be recalled that despite such good nesting $\mathrm{BaFe}_{2} \mathrm{P}_{2}$ is neither superconducting 


\section{$\mathrm{CaFe}_{2} \mathrm{P}_{2}$}
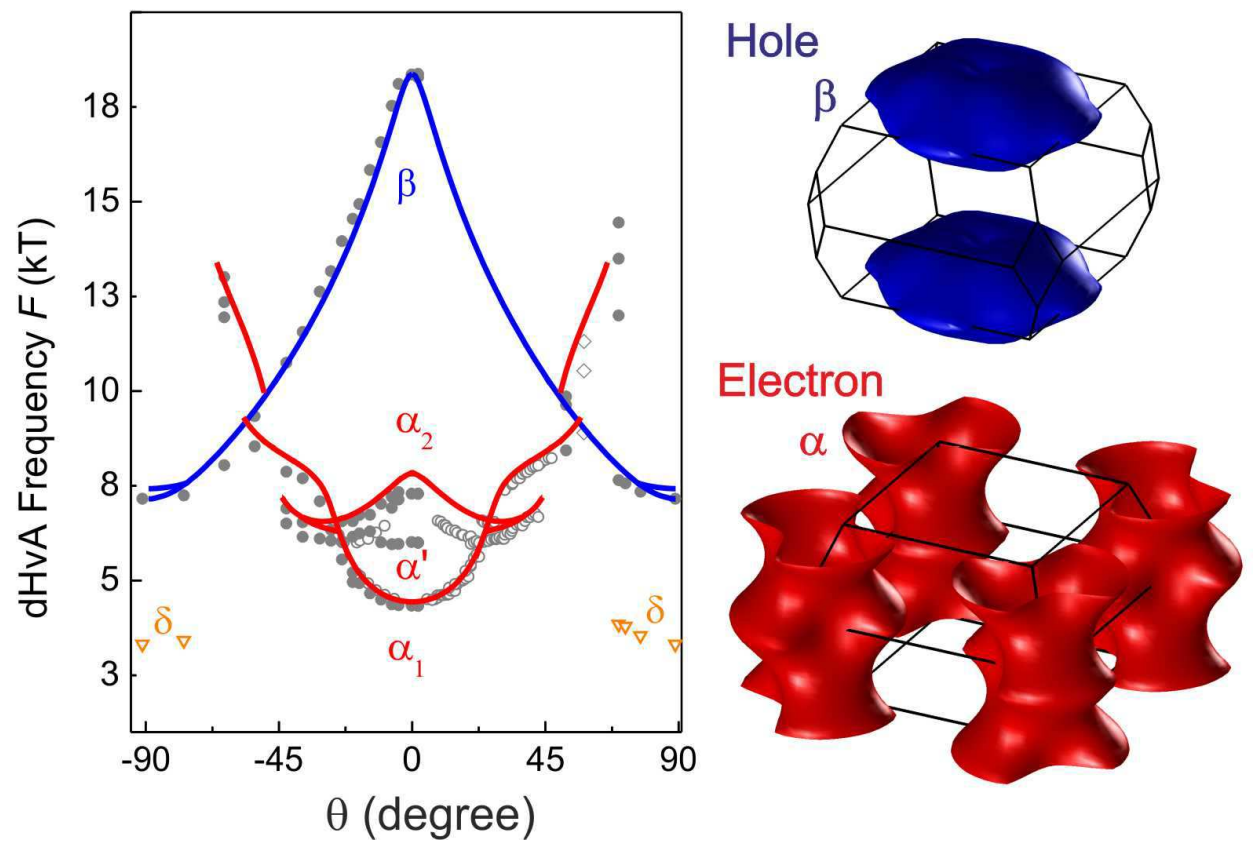

Figure 10. Experimental dHvA frequencies versus magnetic field angle for rotations from $B \| c$ (001) to $B \| a(100)$ for $\mathrm{CaFe}_{2} \mathrm{P}_{2}$ [37. The electron and hole Fermi surface are shown to the right of the figure. Figure adapted from Ref. 37.

nor magnetic. Hence this is a good example showing that nesting is a necessary but not a sufficient condition for the formation of those ground states. Many body correlation effects which become stronger for the arsenide materials clearly also play a key role.

The band shifts needed to get the DFT calculation to agree with the data for $\mathrm{BaFe}_{2} \mathrm{P}_{2}(+68 /+58 \mathrm{meV}$ for the inner/outer electron sheets and $-113 \mathrm{meV}$ for the inner hole sheet) are almost identical to those for $\mathrm{SrFe}_{2} \mathrm{P}_{2}$. In particular, again it is found that the highly warped $d_{z^{2}}$ section of the outer hole sheet agrees well with the calculation without any shift needed.

Next we turn to $\mathrm{CaFe}_{2} \mathrm{P}_{2}$. Here the dHvA spectra are significantly simpler than the other 122 compounds with far fewer observed frequencies (see Fig. 10). As remarked above (Fig. 7), the Fermi surface of this material is markedly different from the other 122s. The hole Fermi surface has changed from two quasi two dimensional cylinders to a single flat pancake which is depressed close to the $\Gamma-Z$ line. The electron sheets have also become much more warped but still roughly keep their cylindrical shape. For this material, it is found that the DFT calculations agree almost perfectly with the dHvA data without any adjustment of the band energies (to with $\pm 10 \mathrm{meV}$ ). This is an important counter-example to the results found for $\mathrm{LaFePO}, \mathrm{BaFe}_{2} \mathrm{P}_{2}$ and $\mathrm{SrFe}_{2} \mathrm{P}_{2}$. It would therefore appear to be clear that the band shifts are not an inherent inaccuracy in the mean-field band-structure but are rather caused by non-mean-field correlation effects which act between almost nested Fermi surfaces. A obvious candidate for this 
are spin-fluctuations.

The mass enhancements found from the dHvA measurements for all three $122 \mathrm{~s}$ plus LaFePO are shown in Table 1. $\mathrm{CaFe}_{2} \mathrm{P}_{2}$ has the smallest enhancements, which are about two times larger than those expected from conventional electron-phonon coupling $\left(\lambda_{E P} \simeq 0.23\right)\left[39\right.$. For $\mathrm{SrFe}_{2} \mathrm{P}_{2}$ the enhancements are quite sheet dependent, being only slightly larger than for the Ca compound for most sheets except for the most two dimensional $\alpha$ electron sheets where they are a factor of two larger. $\mathrm{BaFe}_{2} \mathrm{P}_{2}$ again has some sheet dependence and the average $\lambda=0.74$ - about $50 \%$ larger than for $\mathrm{CaFe}_{2} \mathrm{P}_{2}$. A useful check of consistency is to apply the measured mass enhancements for each sheet to the calculated density of states for each sheet and therefore get an estimate of the electronic specific heat. For $\mathrm{SrFe}_{2} \mathrm{P}_{2}$ this gives $\gamma_{E}=10.4(2) \mathrm{mJ} \mathrm{mol}^{-1} \mathrm{~K}^{-2}$, which agrees well with the experimental value of $\gamma_{E}=11.6(2) \mathrm{mJ} \mathrm{mol}^{-1} \mathrm{~K}^{-2}$ [40].

\section{1. $\operatorname{BaFe}_{2}\left(A s_{1-x} P_{x}\right)_{2}$}

Although studies of the non-superconducting end members described above can give valuable insight into the bulk electronic structure of the superconductors, it is clearly preferable to measure the actual superconducting materials themselves. Unfortunately, in the main, the condition that the samples must be free of disorder means that it is not possible to observe quantum oscillations in heavily doped superconducting samples. This is compounded by the fact that often the highest $T_{c}$ samples also have very high $H_{c 2}$ values and so the normal state cannot be accessed. LaFePO and $\mathrm{KFe}_{2} \mathrm{As}_{2}$ [41] are rare examples where superconductivity occurs in the (close to) stoichiometric composition and dHvA oscillations are observable. However, it is unclear whether these low $T_{c}$ materials are representative of the higher $T_{c}$ pnictides. Fortunately, there is one exception to this. In the isovalently substituted $\mathrm{BaFe}_{2}\left(\mathrm{As}_{1-x} \mathrm{P}_{x}\right)_{2}$ series the residual resistance, and hence scattering, remains low even as the maximum $T_{c}$ is approached $\left(T_{c}^{\max }=30 \mathrm{~K}\right.$ for $\left.x=0.33\right)$ [42]. In this material, and other pnictides, there is clear evidence that the transport properties develop 'non-Fermi liquid' properties as the maximum $T_{c}$ is approached. The resistivity follows a power law as a function of temperature, $\rho \propto T^{n}$ where the exponent $n$ evolves from its conventional Fermi liquid value of 2 for the overdoped phosphide to close to 1 at optimal doping. Such behaviour is strongly reminiscent of the high $T_{c}$ cuprate superconductors [43] and heavy Fermions in the vicinity of a quantum critical point [44]. In $\mathrm{BaFe}_{2}\left(\mathrm{As}_{1-x} \mathrm{P}_{x}\right)_{2}$ the highest $T_{c}$ occurs approximately where the SDW order temperature extrapolates to zero and hence is likely strongly influenced by the presence of a quantum critical point [45].

Using pulsed magnetic fields up to $60 \mathrm{~T}$ dHvA oscillations were observed in the $\mathrm{BaFe}_{2}\left(\mathrm{As}_{1-x} \mathrm{P}_{x}\right)_{2}$ series for $x$ down to 0.42 , where $T_{c}$ has reached $25 \mathrm{~K}$ which is $\sim 80 \%$ of the maximum [10]. The quite remarkable fact that oscillations could still be seen in such a heavily substituted composition implies that As/P cross substitution induces very little disorder on the conducting Fe plane. The observed frequencies were identified as coming from the electron sheets, and for the lower values of $x$ (higher $T_{c}$ ) only a single 


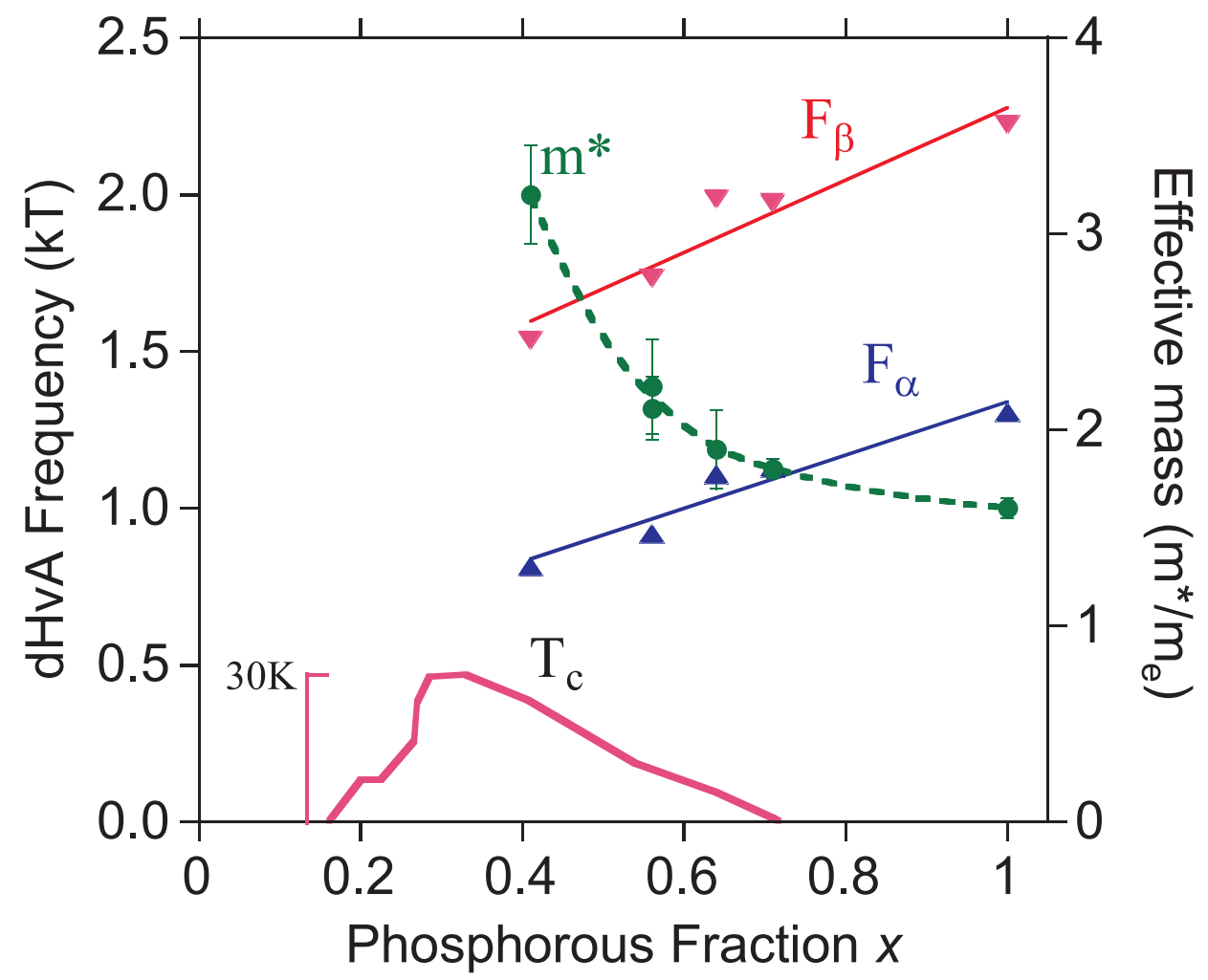

Figure 11. Experimental dHvA frequencies for the $\alpha$ and $\beta$ electron orbits of $\mathrm{BaFe}_{2}\left(\mathrm{As}_{1-x} \mathrm{P}_{x}\right)_{2}$ versus the phosphorous content $x$. Where more than one $\alpha / \beta$ frequency was observed the average frequency/mass is plotted here. The $x$ dependence of the effective mass $m^{*}$ of the $\beta$ orbits and $T_{c}$ is also shown. Figure adapted from Ref. [10, 38. The more accurate value of $m^{*}$ from Ref. 38. for $x=1$ is plotted in preference to that in Ref. [10].

frequency from each electron band was discernable. In the higher $T_{c}$ samples oscillations were only observable at the highest fields and hence the Fourier peaks are too broad to see the individual peaks.

Two important results were found. First it was observed that both the $\alpha$ and $\beta$ frequencies decrease markedly with decreasing $x$ (see Fig. 11). No such decrease is expected from the DFT band structure calculations. Second, the effective mass increases strongly as the maximum $T_{c}$ is approached. It is likely that both these changes are caused by the increase in correlations (spin fluctuations) as the SDW ordered state is approached. It is not clear from the available data whether the increase in effective mass is a divergence associated with a possible quantum critical point or rather just a general increase with decreasing $x$. Unfortunately, no data is yet available for the values of $x$ below the critical value.

Recently Analytis et al. [46] reported measurements for the $x=0.63$ with $T_{c}=7 \mathrm{~K}$ where orbits on the smaller hole sheet were observed. The frequency of these orbits were found to correspond to almost the same cross-sectional area as the smaller electron pocket, very similar to that found for the $x=1$ composition described above. Hence it 
would appear that the relative size of the electron and hole sheets remain remarkably constant as a function of $x$. As the electron sheets shrink with decreasing $x$ the hole bands shrink by a similar amount without any major change in topology. This is supported by recent ARPES results for the $x=0.38$ composition [47] which show that in this close to optimal doped composition the shape of the Fermi surfaces closely resemble the end member $\mathrm{BaFe}_{2} \mathrm{P}_{2}$.

A semi quantitative model of the electron / hole pocket shrinking of the quasinested electron-hole Fermi surfaces of iron-pnictides has been suggested by Ortenzi et al. [48]. Here scattering caused by coupling of the itinerant electrons to a bosonic spin fluctuation mode (assumed to be Einstein-like) causes both electron and hole pockets to shrink and also the effective mass (and $T_{c}$ ) to increase. This was applied to a simplified model of the LaFePO Fermi surface and reasonable quantitative agreement was found between the magnitude of the energy shifts (which in this model determines the strength of the scattering potential) and the mass enhancement and even $T_{c}$. It should be noted however that taken at face value this model would predict low-energy correlation-driven renormalisation of the band energies (i.e., kinks in $\varepsilon(\boldsymbol{k})$ close to the Fermi level), whereas a comparison of the $\mathrm{dHvA}$ data to ARPES suggests the renormalisation occurs over a much wider energy range. It would however be intriguing to see if an extension of this model could explain the correlations between band energy shifts, effective masses and $T_{c}$ in the $\mathrm{BaFe}_{2}\left(\mathrm{As}_{1-x} \mathrm{P}_{x}\right)_{2}$ series. This could prove to be a decisive test of the spin fluctuation pairing model in the iron-pnictides.

\section{Summary and Conclusions}

Quantum oscillation measurements have proven to be a very valuable probe of the bulk electronic structure of iron-based superconductors. Combined with first principle electron structure calculations a very detailed model of the Fermi surface and the quasiparticle dynamics can be obtained. This review has shown that density functional theory calculations provide a good starting point. Using $k$-independent shifts to the band energies the band structure calculations can be fitted to the data using very few parameters. These shifts appear to originate from strong electron correlations and are present even for the non-superconducting, non-magnetic phosphides. Importantly, it is found that only those parts of the Fermi surface where there is electron-hole quasi-nesting are shifted in energy. For $\mathrm{CaFe}_{2} \mathrm{P}_{2}$ no shifting is necessary. In the $\mathrm{BaFe}_{2}\left(\mathrm{As}_{1-x} \mathrm{P}_{x}\right)_{2}$ series the shifting increases as the SDW phase transition is approached and is accompanied by a strong increase in the effective mass. It is hoped that in the near future, more sophisticated first principle calculations which go beyond the meanfield level, such as dynamical mean field theory, may explain these results and lead to new insights into the physic of iron-based superconductors. 


\section{Acknowledgements}

I am indebted to my many colleagues who have contributed to many of the experiments described in this review. In particular, I would like to acknowledge the contributions of Amalia Coldea, Jon Fletcher, Brendan Arnold, Caroline Andrew, Ali Bangura, Patrick Rourke (Bristol), James Analytis, Ian Fisher (Stanford), Yuji Matsuda, Takasada Shibauchi, Shigeru Kasahara (Kyoto), Cyril Proust, David Vignolles, and Baptiste

Vignolle (Toulouse) with whom I personally have worked most closely. This work was supported by the UK EPSRC.

\section{References}

[1] Singh D J and Du M H 2008 Phys. Rev. Lett. 100237003

[2] Mazin I I, Singh D J, Johannes M D and Du M H 2008 Phys. Rev. Lett. 101057003

[3] Kuroki K, Onari S, Arita R, Usui H, Tanaka Y, Kontani H and Aoki H 2008 Phys. Rev. Lett. 101 087004

[4] Chubukov A V, Efremov D V and Eremin I 2008 Phys. Rev. B 78134512

[5] Cvetkovic V and Tesanovic Z 2009 EPL 8537002

[6] Kamihara Y, Hiramatsu H, Hirano M, Kawamura R, Yanagi H, Kamiya T and Hosono H $2006 \mathrm{~J}$. Am. Chem. Soc. 12810012

[7] Kamihara Y, Watanabe T, Hirano M and Hosono H 2008 J. Am. Chem. Soc. 130 3296-3297

[8] Mazin I I and Schmalian J 2009 Physica C 469 614-627

[9] Shoenberg D 1984 Magnetic Oscillations in Metals (Cambridge: Cambridge University Press)

[10] Shishido H, Bangura A F, Coldea A I, Tonegawa S, Hashimoto K, Kasahara S, Rourke P M C, Ikeda H, Terashima T, Settai R, Onuki Y, Vignolles D, Proust C, Vignolle B, Mccollam A, Matsuda Y, Shibauchi T and Carrington A 2010 Phys. Rev. Lett. 104057008

[11] Rotter M, Tegel M, Johrendt D, Schellenberg I, Hermes W and Pöttgen R 2008 Phys. Rev. B 78 020503

[12] Tanatar M A, Blomberg E C, Kreyssig A, Kim M G, Ni N, Thaler A, Bud'ko S L, Canfield P C, Goldman A I, Mazin I I and Prozorov R 2010 Phys. Rev. B 81184508

[13] Chu J H, Analytis J G, Press D, De greve K, Ladd T D, Yamamoto Y and Fisher I R 2010 Phys. Rev. B 81214502

[14] Chu J H, Analytis J G, De greve K, Mcmahon P L, Islam Z, Yamamoto Y and Fisher I R 2010 Science 329 824-826

[15] Yi M, Lu D H, Analytis J G, Chu J H, Mo S K, He R H, Hashimoto M, Moore R G, Mazin I I, Singh D J, Hussain Z, Fisher I R and Shen Z X 2009 Phys. Rev. B 80174510

[16] Analytis J G, Mcdonald R D, Chu J H, Riggs S C, Bangura A F, Kucharczyk C, Johannes M and Fisher I R 2009 Phys. Rev. B 80064507

[17] Sebastian S E, Gillett J, Harrison N, Lau P H C, Singh D J, Mielke C H and Lonzarich G G 2008 J. Phys. Cond. Matt. 20422203

[18] Harrison N, Mcdonald R D, Mielke C H, Bauer E D, Ronning F and Thompson J D $2009 \mathrm{~J}$. Phys.-Condes. Matter 21322202

[19] Note that Terashima et al., suggest that the second of these frequencies is actually the second harmonic of the first 20

[20] Terashima T, Kurita N, Tomita M, Kihou K, Lee C H, Tomioka Y, Ito T, Iyo A, Eisaki H, Liang T, Nakajima M, Ishida S, Uchida S, Harima H and Uji S ArXiv:1103.3329

[21] Coldea A I, Fletcher J D, Carrington A, Analytis J G, Bangura A F, Chu J H, Erickson A S, Fisher I R, Hussey N E and Mcdonald R D 2008 Phys. Rev. Lett. 101216402 
[22] Hamlin J J, Baumbach R E, Zocco D A, Sayles T A and Maple M B 2008 J. Phys. Cond. Mat. 20365220

[23] Mcqueen T M, Regulacio M, Williams A J, Huang Q, Lynn J W, Hor Y S, West D V, Green M A and Cava R J 2008 Phys. Rev. B $\mathbf{7 8} 024521$

[24] Carrington A, Coldea A I, Fletcher J D, Hussey N E, Andrew C M J, Bangura A F, Analytis J G, Chu J H, Erickson A S, Fisher I R and Mcdonald R D 2009 Physica C 469 459-468

[25] Sugawara H, Settai R, Doi Y, Muranaka H, Katayama K, Yamagami H and Onuki Y 2008 J. Phys. Soc. Jpn. $\mathbf{7 7} 113711$

[26] Yamaji K 1989 J. Phys. Soc. Jpn. 581520

[27] Bergemann C, Mackenzie A P, Julian S R, Forsythe D and Ohmichi E 2003 Adv. Phys. 52 639-725

[28] Bangura A F, Rourke P M C, Benseman T M, Matusiak M, Cooper J R, Hussey N E and Carrington A 2010 Phys. Rev. B $\mathbf{8 2} 140501$

[29] Rourke P M C, Bangura A F, Benseman T M, Matusiak M, Cooper J R, Carrington A and Hussey N E 2010 New J. Phys. 12105009

[30] Blaha P, Schwarz K, Madsen G K H, Kvasnicka D and Luitz J 2001 WIEN2K, An Augmented Plane Wave + Local Orbitals Program for Calculating Crystal Properties (Karlheinz Schwarz, Techn. Universität Wien, Austria) iSBN 3-9501031-1-2

[31] Boeri L, Dolgov O V and Golubov A A 2008 Phys. Rev. Lett. 101026403

[32] Jiang S, Xing H, Xuan G, Wang C, Ren Z, Feng C, Dai J, Xu Z and Cao G 2009 J. Phys. Cond. Mat. 21382203

[33] Shi H L, Yang H X, Tian H F, Lu J B, Wang Z W, Qin Y B, Song Y J and Li J Q 2010 J. Phys. Cond. Mat. 22125702

[34] Sefat A S, Jin R, McGuire M A, Sales B C, Singh D J and Mandrus D 2008 Phys. Rev. Lett. 101 117004

[35] Alireza P L, Ko Y T C, Gillett J, Petrone C M, Cole J M, Lonzarich G G and Sebastian S E 2009 J. Phys. Cond. Mat. 21012208

[36] Analytis J G, Andrew C M J, Coldea A I, Mccollam A, Chu J H, Mcdonald R D, Fisher I R and Carrington A 2009 Phys. Rev. Lett. 103076401

[37] Coldea A I, Andrew C M J, Analytis J G, McDonald R D, Bangura A F, Chu J H, Fisher I R and Carrington A 2009 Phys. Rev. Lett. 103026404

[38] Arnold B, Kasahara S, Coldea A, Terashima T, Matsuda Y, Shibauchi T and Carrington A 2011 Phys. Rev. B x x

[39] Yildirim T 2009 Physica $C 469$ 425-441

[40] Andrew C.M.J., Ph.D. thesis, University of Bristol, (2011)

[41] Terashima T, Kimata M, Kurita N, Satsukawa H, Harada A, Hazama K, Imai M, Sato A, Kihou K, Lee C H, Kito H, Eisaki H, Iyo A, Saito T, Fukazawa H, Kohori Y, Harima H and Uji S 2010 J. Phys. Soc. Jpn. 79053702

[42] Kasahara S, Shibauchi T, Hashimoto K, Ikada K, Tonegawa S, Okazaki R, Shishido H, Ikeda H, Takeya H, Hirata K, Terashima T and Matsuda Y 2010 Phys. Rev. B 81184519

[43] Kubo Y, Shimakawa Y, Manako T and Igarashi H 1991 Phys. Rev. B 43 7875-7882

[44] Custers J, Gegenwart P, Wilhelm H, Neumaier K, Tokiwa Y, Trovarelli O, Geibel C, Steglich F, Pepin C and Coleman P 2003 Nature 424 524-527

[45] Dai J H, Si Q M, Zhu J X and Abrahams E 2009 Proc. Natl. Acad. Sci. 106 4118-4121

[46] Analytis J G, Chu J H, McDonald R D, Riggs S C and Fisher I R 2010 Phys. Rev. Lett. 105 207004

[47] Yoshida T, Nishi I, Ideta S, Fujimori A, Kubota M, Ono K, Kasahara S, Shibauchi T, Terashima T, Matsuda Y, Ikeda H and Arita R 2011 Phys. Rev. Lett. 106117001

[48] Ortenzi L, Cappelluti E, Benfatto L and Pietronero L 2009 Phys. Rev. Lett. 103046404 